\title{
On Integral Manifolds for Leibniz Algebras
}

\author{
Juan Monterde ${ }^{1}$ and Fausto Ongay ${ }^{2}$ \\ ${ }^{1}$ Departament de Geometria i Topologia, Universitat de València, Burjassot, 46100 València, Spain \\ ${ }^{2}$ CIMAT, Jalisco S/N, Valenciana, 36240 Guanajuato, 36240 GTO, Mexico
}

Correspondence should be addressed to Fausto Ongay; ongay@cimat.mx

Received 1 November 2013; Revised 27 February 2014; Accepted 3 March 2014; Published 24 April 2014

Academic Editor: Pu Zhang

Copyright (C) 2014 J. Monterde and F. Ongay. This is an open access article distributed under the Creative Commons Attribution License, which permits unrestricted use, distribution, and reproduction in any medium, provided the original work is properly cited.

We discuss several partial solutions to the so-called "coquecigrue problem" of Loday; these solutions parallel, but also generalize in several directions, the classical Lie group-Lie algebra correspondence. Our study highlights some clear similarities between the split and nonsplit cases and leads us to a general unifying scheme that provides an answer to the problem of the algebraic structure of a coquecigrue.

\section{Introduction}

As is now well known, Leibniz algebras are a noncommutative, or rather, non-anti-symmetric, generalization of Lie algebras. The so-called "coquecigrue problem" was proposed by J. L. Loday as an analogue for these algebras of Lie's third fundamental theorem: given a finite dimensional Leibniz algebra, look for a manifold possessing an algebraic structure such that its linearization yields the original Leibniz algebra. Ideally, this algebraic structure would be a binary operation, but because there is no formal, precise, definition of what a coquecigrue is, this has proven to be a rather difficult question. To what extent this program can be fulfilled is, as of now, still not entirely clear, but we will describe here what we believe is the right approach.

A first hint at a general answer to this problem first appeared in [1], where it was shown that any Leibniz algebra can in a sense be "integrated," by the judicious choice of a so-called Lie rack, stemming from a construction due to Fenn and Rourke. But this was considered unsatisfactory, because for Lie algebras this does not, in general, give the corresponding Lie group(s), a condition that is normally regarded as a critical property of the solution.

In the same paper it was also argued that, again via an appropriate rack, an answer for split Leibniz algebras can be given in terms of digroups. This is now generally regardedincluding ourselves-as providing a reasonable solution to the coquecigrue problem for this kind of algebras, since the procedure used to obtain a Leibniz algebra from a digroup is in all respects identical to the one used to derive the Lie algebra of a Lie group; in particular, for Lie algebras in principle this reduces to the standard solution given by Lie's theorem.

Nevertheless, because the splittings of a Leibniz algebra are not necessarily unique, this opens up questions of uniqueness of these integral manifolds that in a sense are even more delicate than the standard situation in the classical Lie theory, where groups that are locally but not globally diffeomorphic have the same algebra.

One of our main objectives here (Section 3 ) is therefore to analyze this problem. More precisely, we will see two things: First, we will show that even for the case of Lie algebras, by means of digroups one can obtain valid solutions, different to the classical ones. To our knowledge, this is a new result on the integration of Lie algebras, and in particular, this shows that there can be no unique solution to the problem of integrating a Leibniz algebra in its original form, without some further assumptions about the nature of the expected integral manifold. Second, that the nonuniqueness of solutions extends in another way, by stating explicit homological conditions for obtaining possible integral manifolds for split algebras that are not direct products as digroups are.

In another direction, since the digroup construction does not work for nonsplit Leibniz algebras, a different approach 
is required here. The best results to date for this case were given in the Ph.D. thesis of Covez, [2], who uses some cohomology groups associated with the Leibniz algebra to devise an integration procedure for arbitrary algebras. His solution is also in terms of racks; however, by construction, his results are only local, as a condition of simple connexity of an underlying Lie group is required.

With this in mind, in Section 4 we analyze, from a different point of view, the geometric construction of integral manifolds for a special class of nonsplit Leibniz algebras, whose bracket is a derived one in the sense of [3]. For these particular algebras we can give a more direct construction of an integral manifold than the one in Covez's method, again with a rack structure, but the key point of this example is that some strong similarities with the split case can be clearly exhibited.

Then, on the basis of the different types of solutions thus far considered, we reach our final aim in this work, which is to propose what seems to be a good framework for a general geometrical solution to the coquecigrue problem. The construction, given in Section 5, is roughly as follows.

All the integral manifolds for a Leibniz algebra start with the fact that these algebras have some natural quotients that are Lie algebras. Therefore, it is natural to suspect that the integral manifolds will have the structure of a manifold that projects onto a Lie group related to these algebras. Fiber bundles fulfill this requirement but do not posses in general an a priori algebraic structure; hence, we impose the additional condition that these bundles to be endowed with a rack structure. Finally, we impose a compatibility condition expressed as a commutative diagram relating all the pieces of the construction.

In Section 2 we state some prerequisites and notation and introduce some examples; Section 6 contains some final comments and possible future work.

Finally, since this work deals with both algebraic and geometric constructions, we tried to make our presentation rather self-contained, down-to-earth, and based considerably on the concrete examples of Section 2; we feel that this gives a better insight into the nature of the coquecigrue problem, as well as into the characteristics of a definitive solution (such as the one proposed here). In particular, no attempt is made to state the results in the most general possible context, and for the sake of definiteness we only consider finite dimensional real vector spaces.

\section{Split and Derived Leibniz Algebras}

The purpose of this introductory section is mostly to recall some standard and well-known facts about Leibniz algebras, but also to introduce some useful examples. The basic reference here is [1].

Definition 1. A (left) Leibniz algebra is a vector space $L$, provided with a bilinear map, usually denoted by $[\cdot, \cdot]$, satisfying the (left) Leibniz identity:

$$
[X,[Y, Z]]=[[X, Y], Z]+[Y,[X, Z]]
$$

Lie algebras are obtained if the bracket is antisymmetric; this condition being then equivalent to the Jacoby identity, but in general it is a strong generalization.

One can of course define morphisms, ideals, quotients, and so forth, for Leibniz algebras in the usual way, and the most important instance of a quotient in this category naturally occurs when this quotient is a Lie algebra. The minimal ideal for which this holds is the two-sided ideal $S$ generated by the squares $[X, X]$. In fact, it suffices to consider the left ideal generated by the squares, as this is a two-sided ideal, because of the identity $[[X, X], Y]=0$, which holds for any left Leibniz algebra.

On the other hand, as in the Lie case one can define and adjoint mapping by the assignment. For any $X \in L$, let $a d_{X}$ : $L \rightarrow L$ denote the linear map $a d_{X} Y=[X, Y]$. Then, ad : $L \rightarrow \mathfrak{g l}(L)$ is a Leibniz algebra morphism, and therefore $K=$ ker $a d=\{X \mid[X, Y]=0 \forall Y\}$ is also an ideal of $L$. Moreover, by virtue of the identity previously cited $S \subseteq K$, so $L / K$ is also a Lie algebra.

Example 2. A simple class of Leibniz algebras, which are not in general Lie algebras, can be constructed as follows (see e.g., [4]).

Let $V$ be a vector space and $\varphi \in V^{*}$ a nonnull linear functional; on $\operatorname{Mat}_{k}(V)$ this induces a linear transformation (which we also denote by $\varphi$ ), $\varphi: \operatorname{Mat}_{k}(V) \rightarrow \operatorname{Mat}_{k}(\mathbb{R})$, whose $i j$ entry is simply the original functional $\varphi$. Then $L=$ $\operatorname{Mat}_{k}(V)$, together with the bracket

$$
[X, Y]=\varphi(X) Y-Y \varphi(X),
$$

is a Leibniz algebra; for $k \geq 2$ it is not a Lie algebra, since matrix multiplication is not commutative.

Let us next recall the notion of splitting of a Leibniz algebra. For convenience, let us start by describing the general framework, which is as follows.

Suppose $J$ is a two-sided ideal of a Leibniz algebra $L$, and that as vector spaces one decomposes $L=J \oplus H$; then, as a vector space $H \approx L / J$. However, if $X_{i}=j_{i}+h_{i}, i=1,2$ are two vectors, their bracket decomposes as

$$
\left[X_{1}, X_{2}\right]=\left[j_{1}, j_{2}\right]+\left[j_{1}, h_{2}\right]+\left[h_{1}, j_{2}\right]+\left[h_{1}, h_{2}\right]
$$

thus, while the first three summands lie in the ideal $J$, the last term does not necessarily lie in any of the summands and in particular not in $H$. Thus, in general $H$ is not a Leibniz subalgebra, and so in general it cannot be isomorphic to $L / J$; in other words, the exact sequence of Leibniz algebras

$$
0 \longrightarrow J \longrightarrow L \longrightarrow \frac{L}{J} \longrightarrow 0
$$

does not split. (In fact, as is also well known, the study of the obstructions to these splittings is a basic question in homology theory, and Leibniz algebras were introduced by Loday in a homological context.)

But now, an important fact about $K$ is that it is the maximal ideal $J$ with respect to the following property. If we decompose as vector spaces $L=H \oplus J$ and consider two 
vectors decomposed as $X_{i}=h_{i}+j_{i}$, then $J$ is such that the Leibniz bracket decomposes as $\left[X_{1}, X_{2}\right]=\left[h_{1}, j_{2}\right]+\left[h_{1}, h_{2}\right]$. Notice therefore that, if $H$ happens to be a subalgebra, under this condition the first summand determines a left action of $H$ on the ideal $J$ and moreover, if $S \subset J$, this algebra is a Lie algebra, and $J$ is an $H$-module in the Lie category. Such Leibniz algebras have been called demi-semidirect products, and this motivates the following definition.

Definition 3. Let $L$ be a Leibniz algebra, and let the ideals $S$ and $K$ be as above; if $J$ is any ideal such that $S \subseteq J \subseteq K$, then $L$ is said to split with respect to $J$; if (as vector spaces) there is a direct complement to $J, \mathfrak{h}$, that is, a Lie subalgebra of $L$ and such that $J$ is an $\mathfrak{h}$-module.

It should be noticed that in general $\{0\} \neq S \neq K \neq L$ and that almost any possibility thus allowed can occur; moreover, a Leibniz algebra may split over some of the ideals $S \subseteq J \subseteq K$ but not over others or may not split at all.

Example 4. It is rather easy to see that the $\varphi$-algebras split. They do so over the ideal $J=\operatorname{ker} \varphi$. Indeed, if one fixes any $e \in V$ such that $\varphi(e)=1$, and defines $E=\operatorname{Diag}(e, \ldots, e) \epsilon$ $\operatorname{Mat}_{k}(V)$, then for $X \in L, X=(X-\varphi(X) E)+\varphi(X) E$ gives the desired decomposition.

Notice that $J=S$ here, since

$$
\varphi([X, Y])=\varphi(X) \varphi(Y)-\varphi(Y) \varphi(X),
$$

which implies that $\varphi([X, X])=0$. In fact, the map $\varphi$ is the natural projection $L \rightarrow L / S \cong \mathfrak{g l}(V)$.

Moreover, the $\varphi$-algebras also split over the ideal $K=$ ker $a d=S \oplus \mathbb{R} E$; a decomposition, which the reader can easily verify using the formula above, is given by

$$
X=(X-(\varphi(X)-\operatorname{tr}(\varphi(X))) E)+(\varphi(X)-\operatorname{tr}(\varphi(X))) E,
$$

where tr denotes the trace of the matrix.

On the other hand, a class of nonsplit Leibniz algebras, called derived algebras in [3], can be constructed as follows.

Definition 5. Let $\mathfrak{g}$ be a Lie algebra and $\Delta$ a derivation such that $\Delta^{2}=0$; then, the derived Leibniz algebra is the one obtained by defining the bracket $[x, y]_{\Delta}=[\Delta x, y]$.

Verification that this does indeed define a Leibniz algebra is immediate, but the point is that derived algebras are in general neither Lie algebras nor split.

Example 6. Straightforward computations show that for the two-dimensional non-abelian Lie algebra, $\mathbb{R}^{2}=\left\langle e_{1}, e_{2}\right\rangle$ (the braces denoting the linear span) with the Lie bracket defined by $[x, y]=\left(x_{1} y_{2}-x_{2} y_{1}\right) e_{1}$; the map defined by $\Delta x=x_{2} e_{1}$ is an idempotent derivation. Therefore, by defining the bracket $[x, y]_{\Delta}=[\Delta x, y]=x_{2} y_{2} e_{1}$ a Leibniz algebra is obtained, which is nonsplit.

Indeed, here the ideal $K$ is generated by $e_{1}$, and moreover coincides with the ideal $S$, so this algebra could only split over
$K$; however, any subspace $\mathfrak{h}$ complementary to $K$ must be of the form $\mathfrak{h}=\left\langle f=\alpha e_{1}+e_{2}\right\rangle$; computing the bracket on its generator gives $[f, f]=e_{1} \notin \mathfrak{h}$, so that $\mathfrak{h}$ could not be a subalgebra.

\section{Integration for the Split Case}

3.1. Split Leibniz Algebras and Digroups. As mentioned, the relation of the splitting of algebras to the coquecigrue problem was discussed in [1], which we still more or less follow for a while (but see also [5]), and goes through the notion of digroup.

Definition 7. A digroup is a set $D$ provided with two associative operations, $\vdash, \dashv$, satisfying the compatibility relations

$$
\begin{gathered}
x \dashv(y \dashv z)=x \dashv(y \dashv z) ; \quad(x \vdash y) \vdash z=(x \dashv y) \vdash z ; \\
(x \vdash y) \dashv z=x \vdash(y \dashv z) ;
\end{gathered}
$$

and possessing a distinguished bar unit $e$ (meaning that $e+$ $x=x \dashv e=x$ ), and inverses. This means that for every $x \in D$, there exists a unique element $x^{-1}$ such that $x \vdash x^{-1}=x^{-1} \dashv$ $x=e$.

The digroup is a Lie digroup if $D$ is a manifold and the operations involved are smooth.

Example 8. Associated with the $\varphi$-dialgebras, one can construct $\varphi$-digroups. As in Example 2, choose any $e \in V$ such that $\varphi(e)=1$, and define $E=\operatorname{Diag}(e, \ldots, e) \in \operatorname{Mat}_{k}(V)$; then the open set $D=\varphi^{-1}(G L(k, \mathbb{R}))$ inherits the structure of a digroup, where $E$ is the distinguished bar unit and the operations are defined by $X \vdash Y=\varphi(X) Y, X \dashv Y=X \varphi(Y)$ and the inverse of an element $X$ is given by $X^{-1}=\varphi(X)^{-1} E=$ $E \varphi(X)^{-1}$. Clearly, this is a Lie digroup.

For any digroup $D$ these axioms define two subsets: the set of inverses

$$
G=\left\{y \mid \exists x \text { such that } y=x^{-1}\right\},
$$

and the set of bar units

$$
J=\{y \mid y \vdash x=x \dashv y=x, \forall x\} .
$$

The set $G$ turns out to be a group; since both products restricted to it coincide; in particular, we can omit the symbol for the product of two such elements.

One can also define projections

$$
D \longrightarrow G: x \longmapsto e \dashv x ; \quad D \longrightarrow J: x \longmapsto x \dashv x^{-1},
$$

and simple computations show that as a set and as manifold in the Lie case, $D \approx G \times J$.

Now, given a Lie digroup, a Leibniz algebra is obtained as follows. To begin with, define a conjugation or $A d$-action by

$$
y \longmapsto x \vdash y \dashv x^{-1} .
$$

This gives an example of what is sometimes referred to as a pointed rack. 
Definition 9. A pointed rack is a set $Q$, provided with an application $\circ: Q \times Q \rightarrow Q$ and a distinguished point 1 , satisfying

(i) $x \circ(y \circ z)=(x \circ y) \circ(x \circ z)$.

(ii) For all $a, b \in Q$ there is a unique $x$ such that $b \circ x=a$.

(iii) For all $x \in Q, 1 \circ x=x$, and $x \circ 1=1$.

Pointed racks are perhaps most naturally obtained by conjugation on groups, but the point is that, just as in the case of the standard conjugation in Lie groups, a Leibniz bracket is obtained at the tangent space of a Lie digroup at the bar unit, $T_{e} D$, by differentiating twice the rack operation, once with respect to $x$ and once with respect to $y$ (see e.g. [6]).

More explicitly, if $X, Y$ are two tangent vectors at $T_{e} D$, and $x(s), y(t)$ are two curves such that $x(0)=e=y(0)$ and $x^{\prime}(0)=X, y^{\prime}(0)=Y$, their Leibniz bracket $[X, Y]$ can be obtained as

$$
[X, Y]=\left.\frac{\partial^{2}}{\partial s \partial t}\right|_{s=t=0} x(s) \vdash y(t) \dashv x^{-1}(s) .
$$

That this is well defined follows from the fact that the conjugation given by (11) fixes the bar unit $e$. Therefore, by differentiating the $A d$-action with respect to $y$ for fixed $x$, one obtains a map $A d_{x}: T_{e} D \rightarrow T_{e} D$, which is an automorphism, and by allowing $x$ to vary one obtains a map Ad $: D \rightarrow \operatorname{Aut}\left(T_{e} D\right)$. Since this map sends the point $e$ into the identity mapping in $D$, differentiation with respect to $x$ then gives a map ad: $T_{e} D \rightarrow \mathfrak{g l}\left(T_{e} D\right)$, sending $X \rightarrow a d_{X}$, which allows to define the bilinear mapping $[X, Y]=a d_{X} Y$, just as in the Lie case. The digroup conditions then ensure that this is indeed a Leibniz bracket (see e.g., [1] or [4]).

Since this derivation imitates in every respect the construction of Lie algebras from Lie groups, it is naturally a strong indication that digroups should be considered as an acceptable solution to the coquecigrue problem. Nevertheless, as is also well known this works only in the split case; let us now briefly recall why this is so.

The first key step is to observe that the $A d$-action $x \vdash y \dashv$ $x^{-1}$ actually defines a left action of the group $G$ on $J$, with the bar unit $e$ (which is the only bar unit that is also an inverse) as a fixed point; denote this action by $a \cdot \alpha$, for $a \in G, \alpha \in J$.

The digroup $D$ then becomes isomorphic to the digroup $G \times J$ with operations

$$
\begin{gathered}
(a, \alpha) \vdash(b, \beta)=(a b, a \cdot \beta) ; \\
(a, \alpha) \dashv(b, \beta)=(a b, \alpha),
\end{gathered}
$$

and the conjugation is in fact explicitly given by

$$
(a, \alpha) \vdash(b, \beta) \dashv(a, \alpha)^{-1}=\left(a b a^{-1}, a \cdot \beta\right) .
$$

Remark 10. In fact, every digroup arises this way: from a group action, $G \times J \rightarrow J$, with a (not necessarily unique) fixed point. Moreover, one sees from Formula (13) that only one of the products actually involves the action, namely, the one denoted here $\vdash$. The reader might see [5] for more details, just keeping in mind that a different choice of projections was made there, using $x \mapsto x \vdash x^{-1}$ instead of $x \mapsto x^{-1} \dashv x$, which resulted in working with right actions instead of left actions.

At any rate, for Lie digroups, since as a manifold the tangent space at $e$ splits as $T_{e} D=T_{e} G \oplus T_{e} J$, differentiation of Formula (14) exhibits the way $T_{e} J$ becomes a $T_{e} G$-module, giving the desired splitting of $T_{e} D$ as a Leibniz algebra.

Conversely, given a split Leibniz algebra, written as a direct sum $\mathfrak{g} \oplus J$, where $\mathfrak{g}$ is a Lie algebra and the ideal $J$ is regarded as a $\mathfrak{g}$-module, this infinitesimal action can always be integrated to an action of the simply connected group $G$ such that $\operatorname{Lie}(G)=\mathfrak{g}$, so that a digroup $D=G \times J$ is obtained; then the Leibniz algebra structure is recovered from the resulting rack, in the manner already explained (see for instance [1] for more details).

Up to this point we have discussed mostly well-known facts. But now a subtle, yet important, point arises. As mentioned, a split Leibniz algebra might split in different ways, depending on the chosen ideal $J$ and corresponding choice of Lie subalgebra $\mathfrak{g}$. Therefore, for different splittings the digroups associated with such an algebra by the previous construction will not in general be isomorphic as digroups, even locally, for the simple reason that they would not have (even locally) isomorphic groups of inverse elements. The following simple example, already pointed out in [4], illustrates this.

Example 11. An abelian Leibniz algebra $L$, which of course is a Lie algebra, splits over any subspace $J \subset L$. Therefore, we can consider any nontrivial subspace $J$, take any direct complement $\mathfrak{h}$ to $J$, and consider any abelian Lie group $H$ having $\mathfrak{h}$ as Lie algebra. We can then make $H$ act on $J$ trivially and consider the corresponding digroup $D=H \times J$.

Then, the digroup $D$ so defined is certainly not a Lie group, because inversion is not an involution; indeed, the only inverse elements would be those of the form $(h, 0), h \in H$. Nevertheless, its tangent space at $E=(e, 0)$, where $e \in H$ denotes the identity, is endowed by the previous construction with the structure of an abelian Leibniz algebra, therefore, isomorphic to $L$.

Now, while the example of an abelian algebra might seem somewhat artificial, since there is little to check in this case, one can further elaborate on this point, to see that the problem runs deeper.

For this recall that any Lie algebra $\mathfrak{g}$ can be decomposed, although not in a unique way, as a direct sum $\mathfrak{g}=\mathfrak{x} \oplus \mathfrak{h}$, where the radical $\mathfrak{r}$ is the maximal solvable ideal of $\mathfrak{g}$ and the summand $\mathfrak{h}$ is semisimple. This is the so-called Levi (or radical) decomposition of $\mathfrak{g}$.

Now, let $L$ be a Leibniz algebra, let $S$ and $K$ be as before, and let $\mathfrak{g}=L / S$; then, by the isomorphism theorem there is a $1: 1$ correspondence between the ideals $S \subseteq J \subseteq L$ and the ideals of $\mathfrak{g}$. We claim that $K / S$ is contained in $\mathfrak{x}$. Indeed, if $k_{1}+S, k_{2}+S$ are two classes in $K / S$, then $\left[k_{1}+S, k_{2}+S\right]=$ $\left[S, k_{2}\right]+[S, S]=S$, so that $(K / S)^{2}=0$.

This observation then gives two things. 
On the one hand, it indicates that, for a given Leibniz algebra $L$, the obstruction to a splitting is related to the structure of the radical of $\mathfrak{g}=L / S$.

On the other hand, and more important for us here, this implies the following theorem, which is one of our main results.

Theorem 12. Suppose $\mathfrak{j}$ is an abelian ideal of a Lie algebra $\mathfrak{g}$ contained in ker ad that admits a nontrivial complementary subalgebra; then, there is a digroup that is not a Lie group, whose tangent space at the bar unit inherits the structure of the given algebra.

Proof. Indeed, let $\mathfrak{h}$ be a subalgebra such that $\mathfrak{g}=\mathfrak{j} \oplus \mathfrak{h}$ and consider a simply connected Lie group $H$ having Lie algebra $\mathfrak{h}$. Then, the infinitesimal action of $\mathfrak{h}$ on $\mathfrak{j}$ lifts to an action of $H$ on $\dot{j}$, and this yields a digroup that is not isomorphic to a Lie group, since its set of bar units is nontrivial. Nevertheless, its tangent space at $(0, e)$ inherits, by the previous definition of the $A d$-action for digroups, the structure of a Leibniz algebra $\mathfrak{g}$; since $\mathfrak{j}$ is abelian, this Leibniz algebra is easily seen to coincide with the original Lie algebra.

The relevance of this theorem is that it shows that, even for Lie algebras, if we drop the a priori condition of the (local) group structure of the integrating manifold, then the Lie algebra does not in general determine a unique structure. In the wider class of digroups different-and essentially nonequivalent-solutions can be found. The classical Lie theory bypasses this problem, because the group structure is assumed a priori.

Remark 13. As a consequence, in our view these new and somewhat odd nonstandard integral manifolds for the type of Lie algebras considered in the previous theorem should be regarded as the first nontrivial examples of true coquecigrues, the term "coquecigrue," playfully introduced by Loday, referring to some kind of strange mythical beast.

In any case, explicit examples of these highly nonequivalent integral manifolds for non-abelian Lie algebras can be obtained from the Levi decomposition theorem, and this provides nontrivial examples of these coquecigrues; for instance, this is the case where $\mathfrak{j}$ is the radical $\mathfrak{r}$ and this ideal is contained in its centralizer.

Example 14. Consider the reductive Lie algebra $\mathfrak{g l}(n, \mathbb{R})$, where we can take as $\dot{j}$ either the trivial ideal or the 1dimensional center. Two different digroups giving rise to this Leibniz algebra are then $G L(n, \mathbb{R})$ and $S L(n, \mathbb{R}) \times \mathbb{R}$, where the former is the standard solution and is of course a Lie group, but the latter is to be regarded as a nontrivial digroup, with set of bar units $J \cong \mathbb{R}$.

Moreover, since as manifolds both digroups are diffeomorphic, there are no hidden geometrical anomalies that would compel us to disregard the nonstandard solution either.

And this fact actually lies beneath the possibility of splitting a $\varphi$-dialgebra in two different ways discussed in Example 4. The first decomposition, corresponding to the choice of the ideal $\operatorname{ker} \varphi$, results in having $G L(n, \mathbb{R})$ as group of inverses of the associated digroup; the second one, which is related to the ideal ker $a d$, gives a digroup whose group of inverses is $S L(n, \mathbb{R}) \times\{ \pm 1\}$.

3.2. A Generalization of Digroups. A different kind of generalization of the construction of integral manifolds for split Leibniz algebras comes from the observation that the computation of Formula (12) does not require a knowledge of the global structure of $D$, and in particular of its decomposition as a product $G \times J$. The following construction was first presented in [5], and the reader is referred to, for example, [7] for more details on the theory of principal and associated bundles.

Let $G$ be a Lie group, and let $P$ be a $G$-principal bundle over $G$. Then, if $J$ is a left $G$-space with action $\rho$, we can consider a bundle $E$ associated with $P$ via this action; we denote the corresponding projection by $\pi: E \rightarrow G$. Our aim is then to see under what conditions we can define a binary operation $E \times E \rightarrow E:(x, y) \mapsto p \odot q$ that upon differentiation gives a Leibniz algebra structure to $T_{\xi} E$, where $\xi$ is some distinguished point. For if we can accomplish this then $E$ can again be regarded as a possible solution to the coquecigrue problem.

A first problem is that, in contrast to the digroup case, for a general bundle $E$ we do not have a priori the natural choice of a distinguished point $\xi$ at our disposal here, so let us first look for conditions for the existence of such a distinguished point.

Lemma 15. Let $G$ be a Lie group, $P$ a G-principal bundle over a manifold $M$, $J$ a left $G$-space, and $E$ a bundle associated to $P$, with projection $\pi: E \rightarrow M$, via an action with a fixed point $\varepsilon \in J$. Then $E$ admits a natural global section $\sigma_{\varepsilon}$ associated with $\varepsilon$.

Proof. Let $\Psi=(\pi, \psi)$ be any trivialization over an open set $\pi^{-1}(U)$; then $\sigma_{\varepsilon, U}: U \rightarrow E$, given by $\sigma_{\varepsilon, U}(a)=\Psi^{-1}(a, \varepsilon)$, clearly defines a section over $U$. To see that this defines a global section, assume $\Psi_{1}=\left(\pi, \psi_{1}\right), \Psi_{2}=\left(\pi, \psi_{2}\right)$ are two trivializations of the bundle, defined over two open sets, $U_{i}$, $i=1,2$, that are trivializing neighborhoods for the bundle with non-empty intersection, so that on $U_{1} \cap U_{2}$ we have the relation:

$$
\psi_{2}(x)=g_{21}(a) \cdot \psi_{1}(x),
$$

where $g_{i j}$ is a cocycle for the bundle. Then for $a \in U_{1} \cap$ $U_{2}$ we have to show that $\sigma_{\varepsilon, U_{1}}(a)=\sigma_{\varepsilon, U_{2}}(a)$, that is, that $\Psi_{1}^{-1}(a, \varepsilon)=\Psi_{2}^{-1}(a, \varepsilon)$. Since $\Psi_{i}$ are diffeomorphisms, this in turn is equivalent to $\Psi_{2} \circ \Psi_{1}^{-1}(a, \varepsilon)=(a, \varepsilon)$. But

$$
\begin{aligned}
\Psi_{2} \circ \Psi_{1}^{-1}(a, \varepsilon) & =\left(a, \psi_{2} \circ\left(\Psi_{1}^{-1}(a, \varepsilon)\right)\right) \\
& =\left(a, g_{21}(a) \cdot \psi_{1} \circ\left(\Psi_{1}^{-1}(a, \varepsilon)\right)\right) \\
& =\left(a, g_{21}(a) \cdot \varepsilon\right)=(a, \varepsilon),
\end{aligned}
$$

since $\varepsilon$ is a fixed point. The same computations show that, indeed, $\sigma_{\varepsilon}$ is independent of the trivialization. 
Thus, if an action has fixed points, as do the actions required for the digroup construction, the choice of one of them, say $\varepsilon$, determines the distinguished point $\xi=\sigma_{\varepsilon}(e)$ in the bundle. On this basis we can state the next result.

Lemma 16. Let $G$ be a Lie group and let $J$ be a left G-space, with a fixed point $\varepsilon \in J$. Let $P$ be a $G$-principal bundle over $G$ and let $E$ be the bundle associated with $P$ via the corresponding right action, with projection $\pi: E \rightarrow G$. Let $\xi$ be the fixed point in the fiber over the identity element of the group, $e$, given by the natural section determined by $\varepsilon$. Assume moreover that $P$ admits a cocycle satisfying the following condition. For all $a, b \in G$ and $\beta \in J$, there exist transition functions $g_{i j}, \widetilde{g}_{i j}$ such that

$$
g_{i j}(a b) a \cdot \beta=a \widetilde{g}_{i j}(b) \cdot \beta .
$$

Let finally $x=[(a, \alpha)], y=[(b, \beta)] \in E$, so that $\pi(x)=a$, $\pi(y)=b$.

Then $E \times E \rightarrow E:(x, y) \mapsto x \odot y$, given by

$$
x \odot y=\left[\left(a b a^{-1}, a \cdot \beta\right)\right],
$$

is globally well defined and thus gives a binary operation on $E$ that lifts the conjugation action in the base $G$.

Before proceeding with the proof, let us make the following comment, regarding the choice of the operation. This comes of course from the fact that we want to generalize the construction for digroups and so we could also start with (13), thus getting two operations, and therefore something closer to a digroup structure. But this is not necessary for us, because one of the products in a digroup is in a sense trivial, and, as we saw, only the rack structure was relevant for the derivation of the Leibniz algebra structure on $T_{\xi} E$, which is our basic concern here. Thus, all that is needed is the operation $\odot$ to be described by something like (14), at least in a neighborhood of $\xi$.

Proof. Let $\Psi=(\pi, \psi)$ be a trivialization of the bundle, defined on an open neighborhood $U$ of $\xi$, and define the operation $\odot$ as

$$
\Psi(x \odot y)=\left(a b a^{-1}, a \cdot \psi(y)\right) .
$$

Now, since $E$ can be described as equivalence classes of pairs, $(g, \gamma), \gamma \in J, g \in U_{i}$, what we need is to see under what conditions such an operation is well defined. So consider two arbitrary representatives for the classes

$$
\left(a_{1}, \alpha_{1}\right),\left(a_{2} \alpha_{2}\right) \in x, \quad\left(b_{1}, \beta_{1}\right),\left(b_{2}, \beta_{2}\right) \in y,
$$

and let us see when the elements $\left(a_{1} b_{1} a_{1}^{-1}, a_{1} \cdot \beta_{1}\right)$ and $\left(a_{2} b_{2} a_{2}^{-1}, a_{2} \cdot \beta_{2}\right)$ are in the same class. By hypothesis $a_{1}=a_{2}=$ $a$, and $b_{1}=b_{2}=b$; then both points $a \cdot \beta_{1} \sim a \cdot \beta_{2}$, are to be regarded as lying on the fiber over $a b a^{-1}$, but possibly on two distinct trivializing open sets $U_{1}, U_{2}$, so this amounts to show that there exists a transition function $g_{12}$ defined on $U_{1} \cap U_{2}$ such that $a \cdot \beta_{1}=g_{12}(a b) a \cdot \beta_{2}$. But by hypothesis, there exists a transition function $\widetilde{g}_{12}$ such that $\beta_{1}=\widetilde{g}_{12}(b) \cdot \beta_{2}$; acting on this equality by $a$, we get therefore the relation

$$
g_{12}(a b) a \cdot \beta_{2}=a \tilde{g}_{12}(b) \cdot \beta_{2},
$$

as a condition on the associated bundle that would allow a global product to be defined by relation (18).

Finally, it is clear that upon projection the operation $\odot$ corresponds to the conjugation in the group $G: \pi(x \odot y)=$ $a b a^{-1}$.

Turning things around, we can now state the relation to the coquecigrue problem as follows.

Theorem 17. Let $L$ be a split Leibniz algebra and let $G$ be a Lie group associated with the quotient Lie algebra g. Assume that the action associated with the splitting lifts to an action of $G$ on a manifold $J$, of dimension equal to that of $L / \mathfrak{g}$. Let $P$ be any $G$-principal bundle that satisfies the conditions of Lemma 16, and let finally $E$ be an associated bundle constructed via this action.

Then, the associated bundle has the structure of a pointed rack and upon differentiation this structure gives a Leibniz algebra structure to $T_{\xi} E$, isomorphic to that of $L$, and is therefore a solution to the coquecigrue problem.

Proof. The conditions on $P$ and $E$ are not empty, since at least the trivial bundles over the simply connected group of $\mathfrak{g}$ satisfy them, and also we have that $E$ is a manifold of the correct dimension; but it is not required that the bundles be trivial or that the fiber be a vector space. Moreover, by Lemma 16 we can define a binary operation on $E$.

Thus, we have to show that $E$ has the structure of a pointed rack, the distinguished point being of course $\xi$. Notice that if we let $x=[(a, \alpha)], y=[(b, \beta)]$, and $z=[(c, \gamma)]$ be three points in $E$, Lemma 16 allows us to work with representatives of the equivalence classes.

Now, the conditions $x \odot \xi=\xi$ and $\xi \odot x=x$ are immediate

$$
\begin{gathered}
x \odot \xi=\left[\left(\text { sea }^{-1}, a \cdot \epsilon\right)\right]=[(e, \epsilon)]=\xi \\
\xi \odot x=[(\text { eae }, e \cdot \alpha)]=[(a, \alpha)]=x .
\end{gathered}
$$

Then, the second condition for a rack amounts to solving the equation $\left[\left(a b a^{-1}, a \cdot \beta\right)\right]=[(c, \gamma)]$ for $b$ and $\beta$, and so we can set

$$
b=a^{-1} c a ; \quad \beta=a^{-1} \cdot \gamma
$$

Finally,

$$
\begin{aligned}
x \odot(y \odot z) & =\left[\left(a\left(b c b^{-1}\right) a^{-1}, a \cdot(b \cdot \gamma)\right)\right] \\
& =\left[\left(a b c(a b)^{-1},(a b) \cdot \gamma\right)\right]
\end{aligned}
$$

while

$$
\begin{aligned}
(x & \odot y) \odot(x \odot z) \\
& =\left[\left(\left(a b a^{-1}\right)\left(a c a^{-1}\right)\left(a b^{-1} a^{-1}\right)\right),\left(\left(a b a^{-1}\right) \cdot(a \cdot \gamma)\right)\right] \\
& =\left[\left(a b c(a b)^{-1},(a b) \cdot \gamma\right)\right],
\end{aligned}
$$

and thus $E$ has the structure of a pointed rack. 
Now, according to Kinyon's result, the data needed to construct the bundle $E$ also guarantee the existence of a digroup $D$, with underlying group $G$ and with $J$ as its set of bar units, that already gives an integral manifold for $L$. But since in a neighborhood of $\xi$, which can be regarded as a point in both $D$ and $E$, both manifolds are diffeomorphic, and since by the computation done in Lemma 16 the product in $E$ is expressed by formula (19), it is clear that their differentials at $\xi$ coincide. This shows that differentiation of $\odot$ indeed determines a Leibniz algebra isomorphic to $L$ in $T_{\xi} E$.

\section{The Nonsplit Case}

4.1. Derived Algebras and an Exponential Coquecigrue. In this subsection we analyze a rather restricted class of nonsplit algebras, but our objective here is mostly to exhibit a different kind of possible solution to the coquecigrue problem and investigate the common traits between this new construction and what we had in the split case.

So, let $A$ be an associative and unital algebra (i.e., possessing a unit element $E$ ). Since the vector spaces we are considering are finite dimensional, for such algebras the exponential mapping exp : $A \rightarrow A$ is well defined by the usual formula $\exp X=\sum_{n=0}^{\infty} X^{n} / n$ !, because the series converges in any norm. Thus, it makes sense to consider the set $Q=\exp (A)$, and since the derivative of exp at 0 is the identity, this is a diffeomorphism when restricted to suitable neighborhoods of $0 \in A$ and $E \in Q$. For simplicity, in what follows we will further assume that the algebra is such that the exponential mapping is injective.

Now assume that $A$ admits an idempotent derivation, $\Delta^{2}=0$. Then the map $\Delta$ is also a derivation of the associated Lie algebra, with the usual commutator bracket, $[\cdot, \cdot]$, so that a Leibniz algebra can be constructed as in Definition 5. Then we can introduce the following.

Definition 18. The exponential coquecigrue of the Leibniz algebra

$$
L=\left(A,[\cdot, \cdot]_{\Delta}\right)
$$

is the rack structure in $Q$ defined by

$$
x \circ y=\exp \Delta X y(\exp \Delta X)^{-1} .
$$

That this definition makes sense is the content of the following.

Theorem 19. Let $L$ denote the Leibniz algebra constructed from $A$ and $\triangle$ as stated above, and $Q=\exp (A)$. For $x=\exp X$, $y=\exp Y$ define

$$
x \circ y=\exp \Delta X y(\exp \Delta X)^{-1}=\exp \Delta X y \exp (-\Delta X) .
$$

Then $(Q, \circ, E)$ is a pointed rack, such that $T_{E} Q \cong L$ as Leibniz algebras, and is therefore a solution to the coquecigrue problem.

Before proving the theorem, let us establish the following easy lemma.
Lemma 20. Let $A, \Delta$ be as above. Then, for any $X, Y \in A$, $[\Delta X, \Delta Y]=0$. In particular, $\exp \Delta X \exp \Delta Y=\exp \Delta(X+Y)$

Proof. We simply compute

$$
\begin{aligned}
{[\Delta X, \Delta Y] } & =\Delta[\Delta X, Y]-\left[\Delta^{2} X, Y\right] \\
& =\Delta(\Delta[X, Y]-[X, \Delta Y]) \\
& =-\Delta[X, \Delta Y]=-[\Delta X, \Delta Y],
\end{aligned}
$$

and the assertion follows.

Proof of Theorem 19. First we notice that for any $x, y \in Q$ we have indeed $x \circ y \in Q$, since by definition $x \circ y=$ $\exp \Delta X y \exp (-\Delta X)$ and in any associative unital algebra $x \exp Y x^{-1}=\exp \left(x Y x^{-1}\right)$ for any $Y$ and invertible element $x$, so the assertion follows.

Next, we verify the conditions for a pointed rack, taking as distinguished point the unit $E$. Since conditions (ii) and (iii) are simple and direct computations, we will only check (i).

On the one hand, using the lemma we have

$$
\begin{aligned}
x \circ(y \circ z) & =x \circ(\exp \Delta Y z \exp (-\Delta Y)) \\
& =\exp \Delta(X+Y) z \exp (-\Delta(X+Y)) .
\end{aligned}
$$

On the other hand, if we let $b=x \circ y=\exp (\Delta X) y \exp (-\Delta X)$ and write $b=\exp B$ we have that $B=\exp (\Delta X) Y \exp (-\Delta X)$. But $\Delta(\exp \Delta X)=0$, since $\Delta I=0$ and $\Delta(\Delta X)^{2}=\Delta^{2} X \Delta X+$ $\Delta X \Delta^{2} X=0$. Therefore, $\Delta B=\Delta Y$, and so

$$
\begin{aligned}
(x \circ y) \circ(x \circ z) & =b \circ(x \circ z) \\
& =\exp \Delta(B+X) z \exp (-\Delta(X+B)) \\
& =\exp \Delta(X+Y) z \exp (-\Delta(X+Y)),
\end{aligned}
$$

and thus (27) defines a rack structure on $Q$, as claimed.

Moreover, using the chain rule and the fact that $\Delta$ is a linear map, upon derivation the exact same computation as in the Lie algebra case gives as bracket $[\cdot, \cdot]_{\Delta}$, as desired.

Let us now make some important observations regarding this construction.

First, notice that relative to the ideal $J=\operatorname{ker} \Delta$, we have a Lie algebra $\mathfrak{g}=L / J$, underlying this Leibniz algebras. Then, if $G$ is any Lie group having Lie algebra $\mathfrak{g}$, with its exponential mapping $\mathfrak{g} \rightarrow G$, the projection $\Pi: Q \rightarrow G, \Pi(x)=\exp (J+$ $h$ ), where $x=\exp (X)$, and $\pi(X)=J+h$, is a morphism of racks onto $G$ with the standard $A d$-action, since the rack operation corresponds to the standard conjugation of $y$ by $\tilde{x}=\exp (\Delta X)$.

Moreover, the map $\Pi$ is a submersion in some neighborhood of $I$, because if $U$ is any neighborhood of $0 \in \mathfrak{g}$, where exp : $\mathfrak{g} \rightarrow G$ is a diffeomorphism, and $V$ is a neighborhood of $0 \in L$, where exp : $L \rightarrow Q$ is a diffeomorphism, then by a general argument of differential topology $\Pi$ restricted to $\exp \left(\pi^{-1}(U) \cap V\right)$ is a submersion. 
Thus, in a way similar to the split case, the integral manifold constructed for $L$ comes equipped with a (at least local) projection into a Lie group that is a morphism of racks.

The following example further illustrates this.

Example 21. We let $L$ denote the Leibniz algebra of Example 6 and observe that in this case both $J$ and $\mathfrak{g}$ are isomorphic to $\mathbb{R}$ and that moreover, by identifying the basis $\left\{e_{1}, e_{2}\right\}$ with the matrices $\left(\begin{array}{ll}0 & 1 \\ 0 & 0\end{array}\right),\left(\begin{array}{ll}0 & 0 \\ 0 & 1\end{array}\right)$, matrix expressions can be obtained. In particular, we can compute the exponential of elements of $L$ using the standard exponential exp $: \mathfrak{g l}(2, \mathbb{R}) \rightarrow G L(2, \mathbb{R})$. A short computation then shows that in fact

$$
\exp \left(\begin{array}{ll}
0 & x_{1} \\
0 & x_{2}
\end{array}\right)=\left(\begin{array}{cc}
1 & x_{1} \varepsilon\left(x_{2}\right) \\
0 & e^{x_{2}}
\end{array}\right)
$$

where $\varepsilon$ is the analytic function defined by

$$
\varepsilon(t)=\frac{e^{t}-1}{t}
$$

for $t \neq 0$ and $\varepsilon(0)=1$.

The rack structure is then explicitly given by

$$
\exp \left(\begin{array}{ll}
0 & x_{1} \\
0 & x_{2}
\end{array}\right) \circ \exp \left(\begin{array}{ll}
0 & y_{1} \\
0 & y_{2}
\end{array}\right)=\left(\begin{array}{cc}
1 & \left(y_{1}+x_{2} y_{2}\right) \varepsilon\left(y_{2}\right) \\
0 & e^{y_{2}}
\end{array}\right)
$$

Notice in particular that (32) also makes it plain that $Q \approx$ $\mathbb{R}^{+} \times \mathbb{R}$, so it is actually a fiber bundle over the Lie group $\mathbb{R}^{+}$ in this case.

4.2. Relation to the Construction of Covez. As mentioned in the introduction, in [2] a general local solution to the problem of integrating Leibniz algebras was described, and this was done by studying pointed racks and an appropriate cohomology theory for them.

Somewhat more precisely, in Covez's work coquecigrues are obtained as central extensions of Lie groups by modules. The modules are defined by an action $\rho$ of the simply connected Lie group $G_{0}$ associated with the Lie algebra $\mathfrak{g}_{0}=$ $L / K$, whereas before $K=\operatorname{ker} a d$ (note that this ideal is denoted in Covez's paper by $Z_{L}(\mathfrak{g})$, as he uses $\mathfrak{g}$ to denote a Leibniz algebra); the action $\rho$ is given by the lifting of the infinitesimal action of $\mathfrak{g}_{0}$ on the ideal $K$. The extensions are then determined by path integration of a suitable 2-cocycle $\omega$, essentially corresponding to the restriction of the bracket to the complement of the ideal $K$.

Leaving the more technical details aside, let us just add that a key feature of this approach is that, by means of an augmentation process, the theory makes it possible to discriminate among the full category of Lie racks those that allow a good recovery of the Lie algebra-Lie group correspondence, which, as mentioned, is normally considered an essential point for the solution of the coquecigrue problem. Nevertheless, and as the author readily acknowledges, this answer is not complete, because the requisite of simple connexity of the group $G$ makes it clear that the construction is essentially local.
Now, the construction presented in the previous section can be related to Covez's as follows.

For any Lie group $G$ the exponential is a local diffeomorphism, thus, a local inverse (i.e., a logarithmic chart) always exists, $\log : U \subset G \rightarrow \mathfrak{g}$. Therefore, if we start with any Leibniz algebra $L$ and consider the quotient Lie algebra $\mathfrak{g}_{0}=L / K$, we can use a logarithmic chart to pull back the trivial bundle $\pi: L \rightarrow \mathfrak{g}_{0}$, at least to some open set $U \subset G_{0}$. Then we lift the local rack structure of $G_{0}$ to this bundle

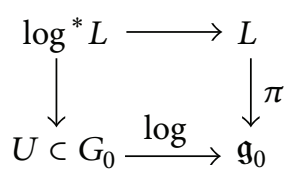

and in this way, a local augmented rack, $\widetilde{Q}=\log ^{*} L \rightarrow U$, is obtained; this should correspond, at least locally, to the construction in [2].

This is the general idea of the relationship between the two approaches, but at this point we need of course to verify several things. In particular, regarding the exponential rack structure in $Q$ previously introduced, to be consistent we need to check that this is indeed the lift of the rack structure in $G$ given by the pullback, so that $Q \cong \widetilde{Q}$ as racks, at least locally.

Now, to check the last assertion, recall first that the pullback bundle is explicitly defined as $\widetilde{Q}=\left(G_{0} \times L\right) / \sim$, where the equivalence relation is given by $\pi(X)=\log g$. Since $\log$ is injective, if we write $\pi(X)=\widetilde{X}$, then we have $g=\exp \widetilde{X}$, and two elements $X, X_{1}$ represent the same class if and only if $X-X_{1} \in K$.

So let $x, y \in Q$; then, given the hypotheses we made for the exponential coquecigrue, $x=\exp X$ and $y=\exp Y$ for unique $X, Y \in L$. Therefore, $x, y$ determine the classes in $\widetilde{Q}$ represented by $(\exp \widetilde{X}, X)$ and $(\exp \widetilde{Y}, Y)$, respectively. Thus, what we need to verify is that if $X_{1}$ and $Y_{1}$ give different representations of the same classes in $\widetilde{Q}$, then $\pi\left(\left[X_{1}, Y_{1}\right]_{\Delta}\right)=$ $\pi\left([X, Y]_{\Delta}\right)$. But, if $X_{1}=X+K_{X}$ and $Y_{1}=Y+X_{Y}$, then

$$
\begin{gathered}
{\left[X_{1}, Y_{1}\right]_{\Delta}=[X, Y]_{\Delta}+\left[X, K_{Y}\right]_{\Delta},} \\
{\left[X, K_{Y}\right]_{\Delta}=\Delta\left[X, X_{Y}\right]-\left[X, \Delta X_{Y}\right]=\Delta\left[X, X_{Y}\right],}
\end{gathered}
$$

because $K_{Y} \in K$ is equivalent to $\Delta X \in Z(A)$ and $\Delta(L) \subset K$, as was to be shown.

Of course, this does not solve the problem for more general algebras, since if we start from a pullback bundle and rack structures, we would need to determine from this data the representation and 2-cocycle required by Covez's theory, and then check that the two constructions are indeed equivalent; in full generality, this is most likely something nontrivial.

Nevertheless, we can show that this makes sense, by explicitly comparing to the Covez data in a simple case. Indeed, consider the exponential coquecigrue of Example 21, where explicit calculations can be done. For this example, the group $G=\mathbb{R}^{+}$is simply connected, and therefore it is actually a simple matter to make a direct connection with Covez's theory (for more details on the following argument, see for instance Example 3.33 in [2]). 
To begin with, we identify the action of $L / K=\mathfrak{g}_{0} \cong$ $\left\langle e_{2}\right\rangle \cong \mathbb{R}$ on $K=\left\langle e_{1}\right\rangle \cong \mathbb{R}$. Since $L / K$ is abelian, it follows that the required action $\rho$ on $K$ is trivial; therefore, it is integrated into the trivial action of $\mathbb{R}$ on itself.

On the other hand, since the only nontrivial bracket is $\left[e_{2}, e_{2}\right]=e_{1}$, it is also clear that the 2-cocycle is $\omega\left(\left(0, x_{2}\right),\left(0, y_{2}\right)\right)=\left(x_{2} y_{2}, 0\right)$. With these considerations, the integration procedure of Covez gives

$$
\int_{\gamma_{a}}\left(\int_{\gamma_{b}} \tau^{2}(\omega)\right)=\omega(a, b) .
$$

Therefore, the local rack structure integrating this Leibniz algebra, according to Covez's method, is

$$
\left(a_{1}, a_{2}\right) \triangleright\left(b_{1}, b_{2}\right)=\left(b_{1}+a_{2} b_{2}, b_{2}\right) .
$$

Now we have to relate both constructions of the coquecigrue, but this amounts to showing that the map defined by Formula (32) is locally a rack isomorphism from $\mathbb{R}^{2} \rightarrow Q$, and this is precisely the content of Formula (34). Indeed, in this simple example the isomorphism is global.

\section{Towards a General Definition of a Coquecigrue}

Drawing from the results discussed for the split case and the construction presented in the previous section, we now describe a possible integration scheme for general Leibniz algebras. Before going into the final definition, let us make some a priori considerations of what such an integral manifold-which as is now customary will usually be called a coquecigrue-should be like.

Suppose we start with a Leibniz algebra $L$ and consider any ideal $J$ such that $S \subseteq J \subseteq K$, so that we have a quotient Lie algebra $\mathfrak{g}=L / J$; let $\pi: L \rightarrow \mathfrak{g}$ denote the corresponding projection. We now observe that we are thereby also given Lie groups $G$, which we could suppose will be connected and simply connected, although this is not essential for our present argument and the corresponding exponential mapping $\mathfrak{g} \rightarrow G$.

Then, collecting these data, we propose that a general scheme for the coquecigrue should not just be a manifold, but a commutative diagram of smooth manifolds and maps of the following type:

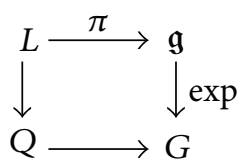

Observe that the arrows linked to the upper right corner summarize the information already known, but also that this is a sort of transposition of the pullback diagram (35), the reason being that the natural map is exp and not log.

Now, to specify what conditions are to be imposed on the remaining objects that complete the diagram, we argue as follows.

First, by definition the manifold $Q$ should have both a distinguished point $\eta$, so that as vector spaces $L \approx T_{\eta} Q$, and an algebraic structure that, upon derivation, yields the Leibniz algebra $L$. From all that has been said, a natural choice for this structure would be that of a pointed rack; however, since an arbitrary rack does not seem appropriate, a key feature of this rack should be that the Leibniz algebra must be obtained from it by a procedure that mimics as much as possible the one for Lie groups, as was the case for instance for digroups.

To explain more precisely what we mean by this, let us consider, after the observation of [1], the objections raised against the rack stemming from the construction by Fenn and Rourke. Recall first that this rack is constructed as follows. Given the Leibniz algebra $(L,[\cdot, \cdot])$, the mapping $a d_{X}: Y \mapsto$ $[X, Y]$ belongs to $\mathfrak{g l}(L)$; since this is a Lie algebra, $\exp \left(a d_{X}\right) \epsilon$ $G L(L)$ is well defined. Therefore, on $L$ itself one can define the rack structure: $X \circ Y=\exp \left(a d_{X}\right)(Y)$; this is the Fenn-Rourke rack structure of $L$.

That this is indeed a rack is essentially due to the relation

$$
A d_{\exp X} Y=\exp \left(a d_{X}\right) Y,
$$

which is valid for any Lie group, applied to the group $G L(L)$, since the crucial rack condition (i) means that

$$
\exp \left(a d_{X}\right) \exp \left(a d_{Y}\right)=\exp \left(\exp \left(a d_{X}\right) a d_{Y}\right) \exp \left(a d_{X}\right),
$$

or, what is the same,

$$
\exp \left(a d_{X}\right) \exp \left(a d_{Y}\right) \exp \left(-a d_{X}\right)=\exp \left(\exp \left(a d_{X}\right) a d_{Y}\right),
$$

which is precisely the relation above for the case of the general linear groups.

Now, the point to be made here is that a key difference between the rack of Fenn and Rourke and the rack obtained by conjugation in the Lie group is that the former does not arise from a "double integration" of the Leibniz algebra structure; indeed, to recover the original Leibniz algebra from this rack, all we need to do is differentiate with respect to the $X$ variable. In other words, by considering the Fenn-Rourke rack we can recover an $A d$-action, but since $\exp X$ is not defined in the Leibniz algebra, in general we do not recover an $A d$-action, so for Lie algebras, unless $a d$ is faithful, we do not expect this rack to be isomorphic to the standard one. (Notice that this problem does not arise for digroups, since in that case we do have an $A d$-action to begin with.)

Therefore, one natural way to codify the desired restriction is to ask that the map $\Pi: Q \rightarrow G$ be a morphism of racks onto the rack given on $G$ by the standard conjugation. This is the second condition that should be imposed on the manifold $Q$ of the diagram.

Moreover, although the above algebraic condition is in a sense the main restriction, there are also topological conditions that are natural. On the one hand, the map $\Psi$ : $L \rightarrow Q$, must be a local diffeomorphism about $0 \in L$; on the other hand, the map $\Pi: Q \rightarrow G$ must be a manifold submersion in a neighborhood of $\eta=\Psi(0)$. But actually, since $Q$ has to be quite homogeneous, so that one might expect to recover a global structure from a local condition, we will strengthen the condition on $\Pi$ to be a global one. 
Thus, gathering all of the above, the definition of a coquecigrue that we propose is as follows.

Definition 22. Let $L$ be a Leibniz algebra and consider any ideal $J$ such that $S \subseteq J \subseteq K$; let the quotient Lie algebra be $\mathfrak{g}=L / J$ and denote the corresponding projection $\pi: L \rightarrow \mathfrak{g}$. Consider a Lie group $G$ having Lie algebra $\mathfrak{g}$.

An admissible coquecigrue for $L$ over $G$ for this data is a commutative diagram of the following type:

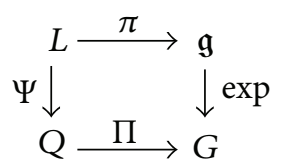

where $Q$ is a fiber bundle over the group $G$, together with a pointed rack structure with distinguished point $\eta ; \Psi: L \rightarrow$ $Q$ is a local diffeomorphism about $0 \in L$, such that $\Psi(0)=$ $\eta$; and the projection $\Pi$ is a rack morphism into $G$, with its standard rack structure given by conjugation.

Of course, from a geometrical point of view, one would think of the manifold $Q$ as providing the desired integral manifold, so that the integration of $L$ is given by the map $\Psi$, just as exp provides the integration of $\mathfrak{g}$, but the algebraic structure of $Q$ needs to be specified by the remaining elements of the diagram. It is also plain that local definitions can be stated, replacing the Lie group $G$ and the Lie rack $Q$ by local ones.

Moreover, notice that the exponential coquecigrues discussed before, as well as digroups, fit well into this scheme and even Covez's construction has these properties too; the bundle being trivial in the last two cases.

Finally, to address the requirement of a good reduction to the classical construction for the case of Lie algebras, observe that when the Leibniz algebra is the Lie algebra $\mathfrak{g}$ of a Lie group $G$, the classical integration of $\mathfrak{g}$ can be recovered by simply identifying it with a "trivial coquecigrue," where the horizontal arrows are just the identity mappings, or, more generally, where $\pi$ is the identity and the homomorphism $\Pi$ is a covering map.

But for example, if we consider the construction of the rack of Fenn and Rourke for a general Lie algebra, in view of the relation (40), this can also be cast into our scheme, leading to the following diagram:

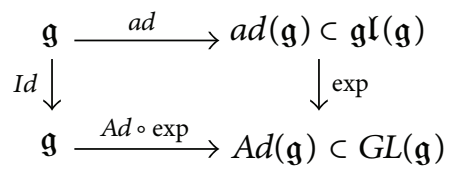

Thus, although this would again be an admissible coquecigrue, since in general ad is not an isomorphism, the coquecigrue associated with the Lie algebra of a group $G$ by this rack would not be associated with the original Lie group, except in those cases where the adjoint group is locally isomorphic to this group, which is alright.

Put another way, in the sense of our definition, in general a Lie algebra $\mathfrak{g}$ could be considered as a coquecigrue over its adjoint group, via the projection $a d$, but one can distinguish between the two integration schemes when they are in fact inequivalent, as desired. And in fact, this allows us to solve the apparently mysterious case of the two nonisomorphic digroups integrating the Lie algebra $\mathfrak{g l}(n, \mathbb{R})$, because $\mathfrak{g l}(n, \mathbb{R}) \cong \operatorname{ad}(\mathfrak{g l}(n, \mathbb{R}))$.

\section{Some Concluding Remarks}

It should be pointed out that there are other approaches to the coquecigrue problem that have appeared in the literature; for instance, an interesting one is given in [8], following ideas of Loday and Pirashvili in [9]. Here the author considers the universal enveloping algebras of Leibniz algebras provided by dialgebras, and then, using a Poincaré-Birkhoff-Witt type theorem, constructs a formal group that integrates the Leibniz algebra. Within this approach, a coquecigrue would be defined as a triple consisting of a Lie group $G$, a right representation $\rho$ of $G$ and a morphism from $\rho$ to the right adjoint representation of $G$, and this looks at first sight quite strong, because it is global and general. But again, this was not considered satisfactory, because in the Lie algebra case it does not give the corresponding Lie group, leading rather only to the adjoint group, but also because it does not in general provide an explicit integrating manifold of the correct dimension.

Our construction is certainly closer in spirit to Covez's, in that we do attempt to obtain such an explicit manifold, but there might also be a relationship to Mostovoy's solution, for the following reason. As mentioned, the rack of Fenn and Rourke also gives a global and general, but equally "unsatisfactory," solution to the coquecigrue problem. However, as can be seen from diagram (44), this rack can also be thought of as a coquecigrue over the adjoint group within our scheme. In fact, for an arbitrary Leibniz algebra $L$, consider the adjoint group of the quotient Lie algebra by the ideal $S$; then we have the following diagram, where $\pi$ and $\Pi$ are now the compositions of the horizontal maps:

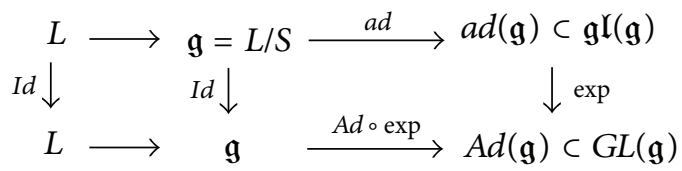

and this diagram essentially contains all the data involved in the solution suggested by [8], which would therefore seem to correspond to one of the solutions allowed by our scheme. But we stress the fact that within our construction we have both: an explicit geometric integral manifold and also a method to discriminate the classical solutions corresponding to the Lie case.

In summary, in our view Definition 22 is an acceptable answer to Loday's original question and thus a good general definition of a coquecigrue. Indeed, an admissible coquecigrue, as defined here, provides an integral manifold for a Leibniz algebra of the correct dimension, as expected from purely geometric considerations, and also fully describes its compatible algebraic structure. 
Granted, the manifold $Q$ does not carry by itself all the structure, but the additional information required is in a definite sense already given. And, while this does allow some nonstandard solutions, it also provides a natural method to recover the correct solution in the classical case, by simply requiring that the bundle projection $Q \rightarrow G$ to be a (local) surjective rack morphism.

Moreover, this also leads to a better understanding of something that was missing in the original coquecigrue problem, in order for it to be well posed; namely, some hypotheses about the structure of the solution, such as the ones in our construction, need to be added. In fact, as our discussion leading to Theorem 12 shows, this is the case even at the Lie algebra level, since applying the classical integration procedure will yield a Lie group only if we specify a priori that this is the algebraic structure required on the integral manifold; otherwise, we have shown that there are instances where other nontrivial solutions, namely, digroups, are possible.

The above remarks also suggest some interesting problems for future work; for instance consider the following.

(i) What are the functorial properties of this construction? Since the admissible coquecigrues are given in terms of commutative diagrams, and in particular this already includes the analogue of the exponential map, it is not unreasonable to expect that a similar analysis to the one of the classical Lie theory can be carried out to a large extent.

(ii) Finding, and if possible classifying, the different admissible coquecigrues associated with a given Lie algebra. As we saw, it should be expected a nonuniqueness problem not only at the level of the global topological structure of the manifold, but also at the level of the algebraic structure. In particular, it would be nice to have something like a 'universal' coquecigrue for a given algebra.

\section{Conflict of Interests}

The authors declare that there is no conflict of interests regarding the publication of this paper.

\section{Acknowledgments}

Fausto Ongay wishes to thank the Departamento de Geometria y Topologia of the Universitat de València, for its kind hospitality while in a sabbatical stay-also supported by a CONACYT grant, when part of this work was done. This work was partially supported by Grant MTM2009-08933 from the Spanish Ministry of Science and Innovation and by CONACYT, Mexico, Project 106923.

\section{References}

[1] M. K. Kinyon, "Leibniz algebras, Lie racks, and digroups," Journal of Lie Theory, vol. 17, no. 1, pp. 99-114, 2007.

[2] S. Covez, "The local integration of Leibniz algebras," Annales de l'Institut Fourier, vol. 63, no. 1, pp. 1-35, 2013.
[3] Y. Kosmann-Schwarzbach, "Derived brackets," Letters in Mathematical Physics, vol. 69, pp. 61-87, 2004.

[4] F. Ongay, " $\varphi$-dialgebras and a class of matrix 'coquecigrues", Canadian Mathematical Bulletin, vol. 50, no. 1, pp. 126-137, 2007.

[5] F. Ongay, On the Notion of Digroup Preprint CIMAT I-10-04 (MB), 2010.

[6] J. J. Duistermaat and J. A. C. Kolk, Lie Groups, Universitext, Springer, Berlin, Germany, 2000.

[7] W. Greub, S. Halperin, and R. Vanstone, Connections, Curvature, and Cohomology, vol. 2 of Mathematics 47-II, Academic Press, New York, NY, USA, 1973.

[8] J. Mostovoy, "A comment on the integration of Leibniz algebras," Communications in Algebra, vol. 41, no. 1, pp. 185-194, 2013.

[9] J. L. Loday and T. Pirashvili, "The tensor category of linear maps and Leibniz algebras," Georgian Mathematical Journal, vol. 5, no. 3, pp. 263-276, 1998. 


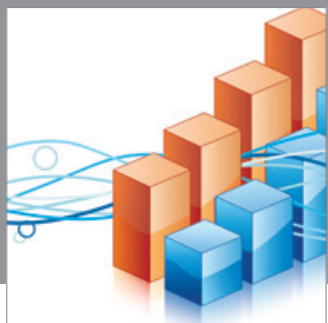

Advances in

Operations Research

mansans

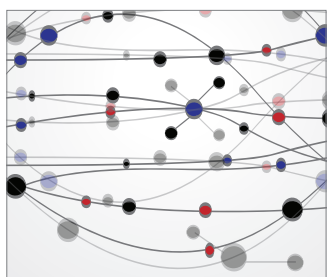

The Scientific World Journal
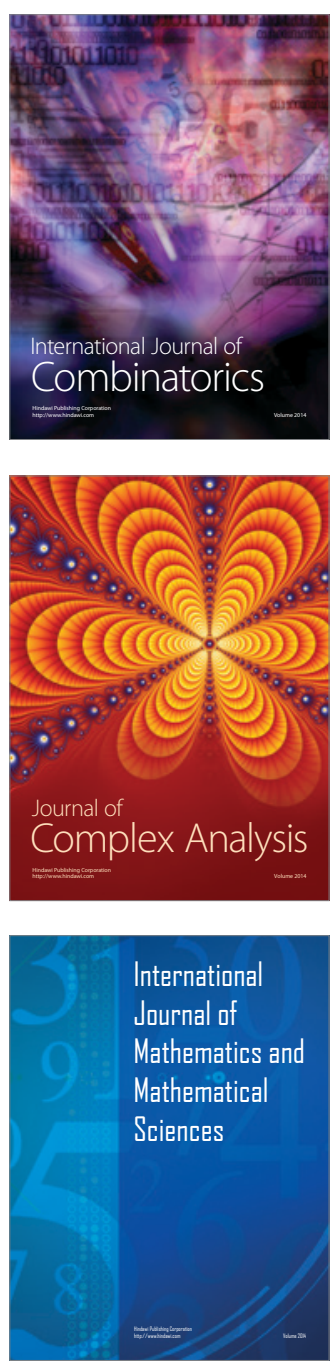
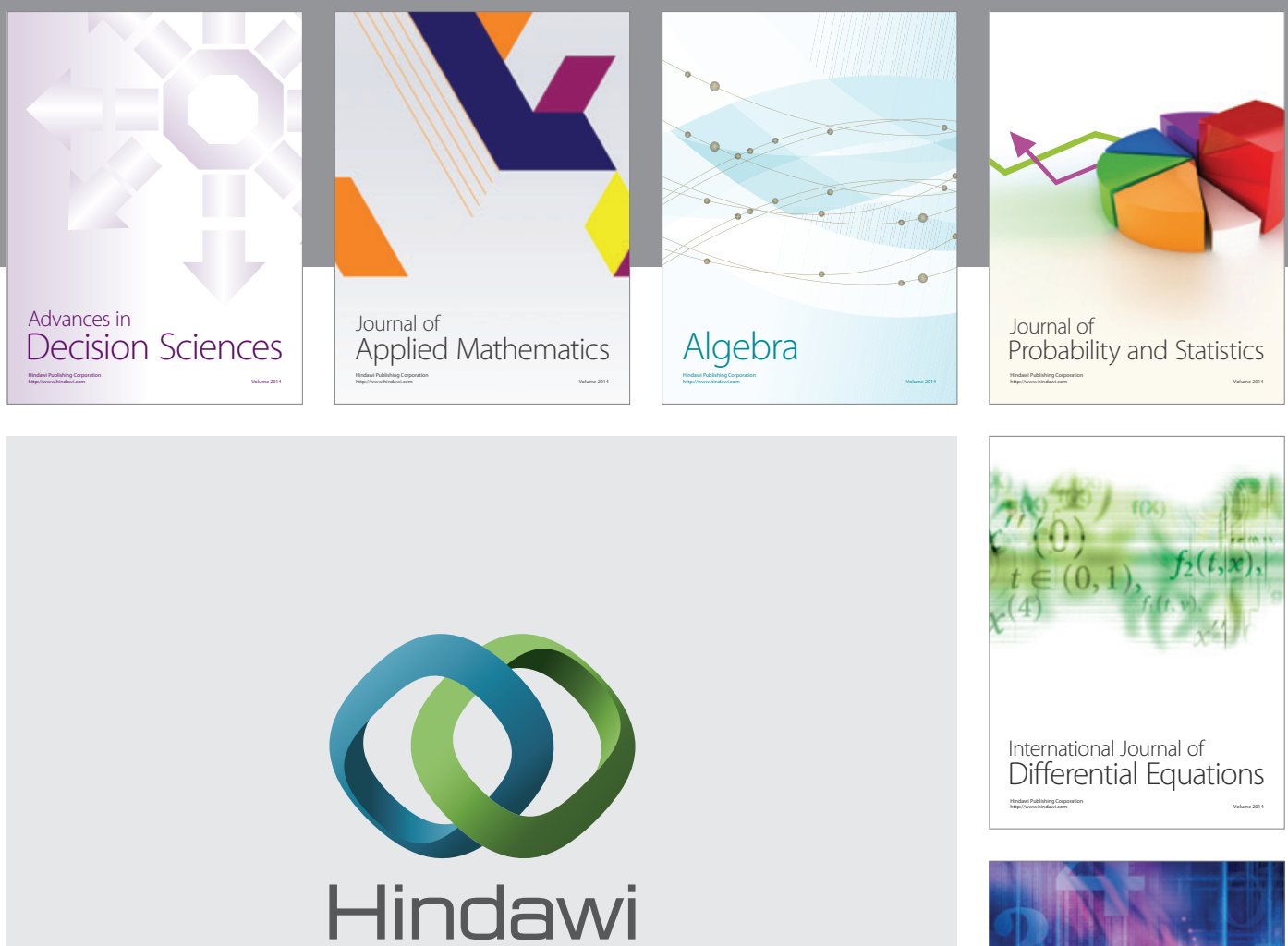

Submit your manuscripts at http://www.hindawi.com
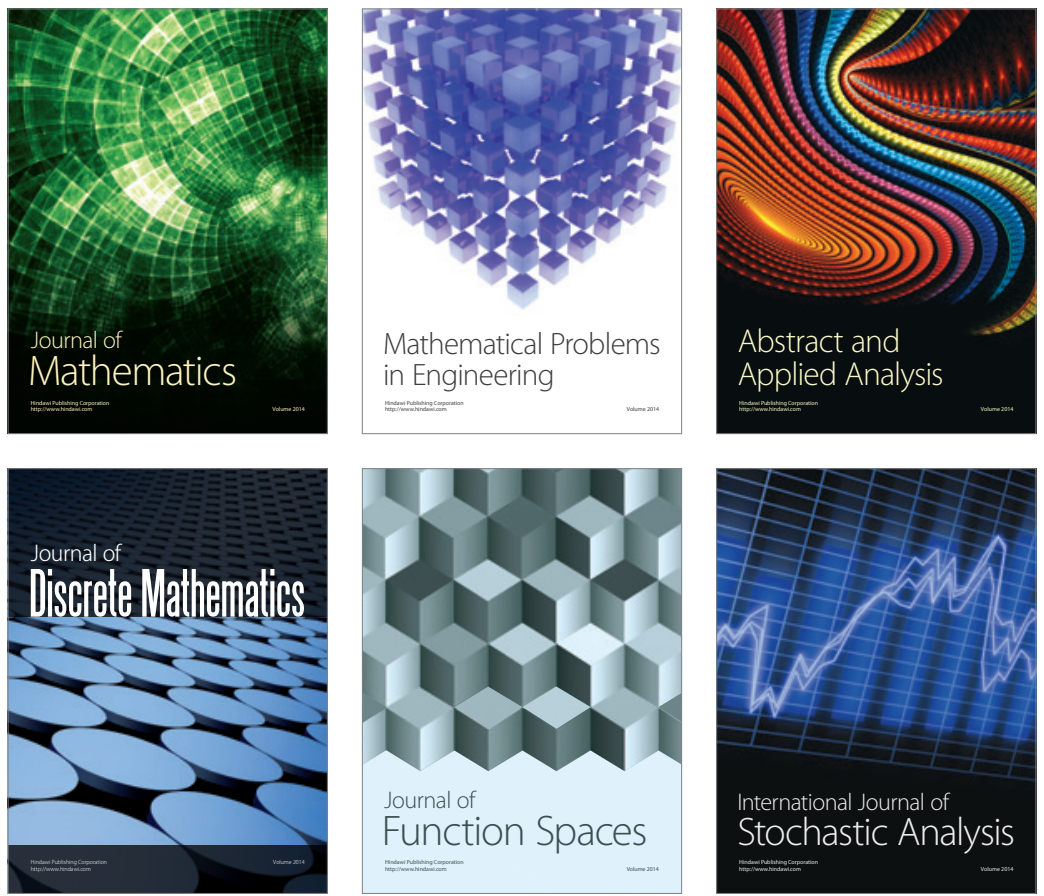

Journal of

Function Spaces

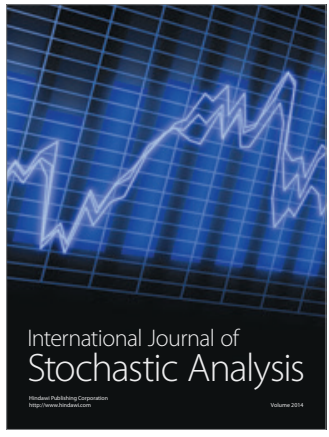

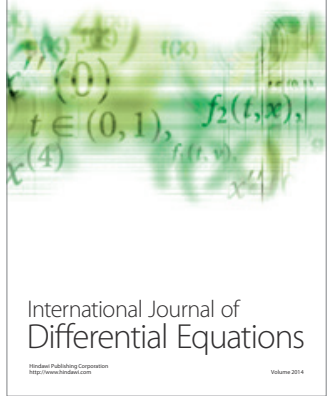
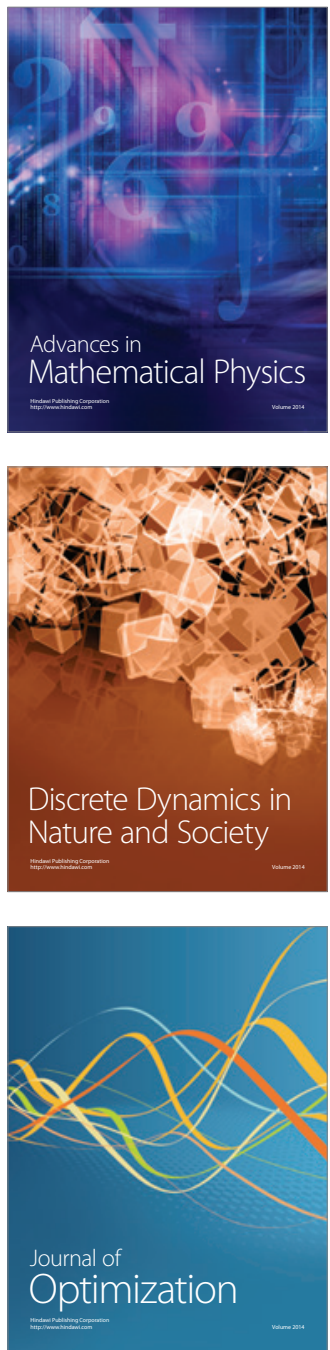